\title{
Biological and biosimilar therapies in inflammatory conditions: challenges for the Central and Eastern European countries
}

\author{
László Gulácsi
}

Published online: 16 May 2014

(c) Springer-Verlag Berlin Heidelberg 2014

This supplement is aimed to give an overview of the current challenges and future prospects of biological and biosimilar drugs in inflammatory conditions in rheumatology (rheumatoid arthritis, ankylosing spondylitis, psoriatic arthritis) gastroenterology (Crohn's disease, ulcerative colitis) and dermatology (psoriasis) with specific focus on a group of Central and Eastern European countries (CEE) such as Bulgaria, the Czech Republic, Hungary, Poland, Romania and Slovakia.

These inflammatory conditions lead to a considerable functional disability, a lowered quality of life and work capacity as well as significant economic burden on patients, families and society.

The first genetically engineered biopharmaceutical drug (called biological) was registered for the treatment of inflammatory conditions 15 years ago and the numbers of these drugs are growing continuously. This group of very effective but very costly new drugs revolutionized the treatment of patients as well as inducing significant development in outcome measures, clinical and financing guidelines and, moreover, speeding up health economic analyses and health technology assessments (HTA) in this field. The first two biosimilar drugs for the treatment of these inflammatory conditions, registered in 2013 by EMA, which are the first marketed in the CEE countries, created a new situation and new challenges for policy makers, funders, professionals, and the patients.

In this supplement we are focusing on economic and financing aspects of biological and biosimilar drugs as well

L. Gulácsi $(\bowtie)$

Department of Health Economics, Corvinus University

of Budapest, Budapest, Hungary

e-mail: laszlo.gulacsi@uni-corvinus.hu as the current stage of HTA and reimbursement decisions in selected CEE countries.

Original research articles supplying inputs for costeffectiveness analyses and dealing with health economic issues are also presented in this supplement from various fields of inflammatory conditions (rheumatoid arthritis, ankylosing spondylitis, Crohn's disease and psoriasis). These are the first studies that provide such data from the CEE region.

The first tumor necrosis factor-alpha inhibitor (hereinafter anti-TNF) drug received its marketing authorisation in Europe (EMA) in 1999 (infliximab), then etanercept (2000), adalimumab (2003) and a further six biological drugs (abatacept, certolizumab pegol, golimumab, rituximab, tocilizumab, ustekinumab) were introduced to the EU market. ${ }^{1}$ These nine biological drugs have altogether 41 indications (30 adult and 11 pediatric, considering disease subtypes and age restrictions) in inflammatory conditions as of March 2014. The two biosimilar drugs (both infliximab) have six adult and two pediatric indications (Table 1).

Clinical evidences confirmed the efficacy and effectiveness of biological drugs in inflammatory conditions. Thus, biologicals became the key treatment options for rheumatoid arthritis, psoriatic arthritis, ankylosing spondylitis, Crohn's disease, ulcerative colitis and psoriasis.

Related to drug registration, there is a considerable harmonisation in Europe. The approval of a pharmaceutical product authorised by the European Medicines Agency (EMA) is binding to all member states.

\footnotetext{
1 Anakinra was also registered by the EMA for the treatment of rheumatoid arthritis but this drug is not prevalent in the CEE countries; therefore, we disregard it in this supplement.
} 


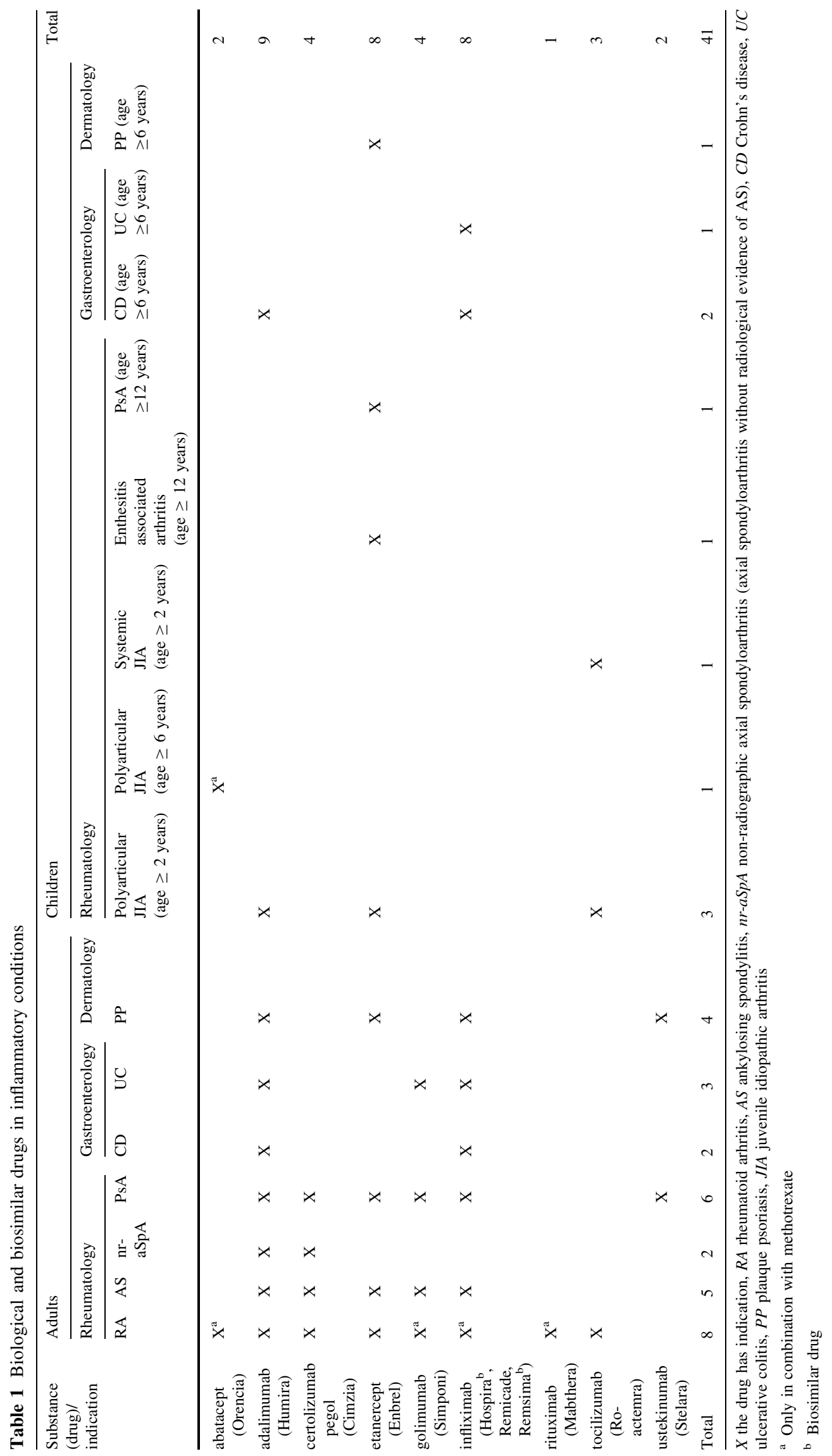


There is a wide European consensus on clinical guidelines based on the best available evidence, which are recommendations on the appropriate treatment and care of people with specific diseases and conditions. Guidelines help healthcare professionals in their work, but they do not replace their expertise and skills [1].

Various professional bodies, for instance the European League Against Rheumatism (EULAR), the European Crohns' and Colitis Organisation (ECCO) and the European Academy of Dermatology and Venerology (EADV) regularly publish their recommendations on the management of inflammatory rheumatic and bowel diseases.

However, although the 28 European Union (EU) member states show uniformity in drug registration and international professional guidelines, in fact, treatment practices are still highly diverse. In countries where biological therapies are not subject to reimbursement, or reimbursed with serious restrictions to a given group of patients with special disease characteristics, the recommendations from professional bodies are less feasible.

In the first decade following the introduction of these new biological drugs, it was mainly patients in high income countries who had access to therapy. Due to the lack of or limited reimbursement, however, only a few patients with inflammatory conditions received biological treatment in the CEE in the first decade of the biological era, compared to high income countries. High prices created a barrier to access for CEE patients, and the utilisation of biological drugs is still lower.

Central and Eastern European countries partly missed the first wave of the diffusion of biologicals, which was very dynamic in most of the high income countries [2]. CEE countries are late adopters of the biological drugs.After 2006-2007, due to the growing number of positive reimbursement decisions, access to biologicals gradually became better and better in CEE. However, today there are still significant variations among countries, even with very similar GDP/capita, and among inflammatory conditions. There are about 0.5 million adults living with inflammatory rheumatic diseases (rheumatoid arthritis, ankylosing spondylitis, psoriatic arthritis) in the six CEE countries, and the number of patients treated with biologicals is estimated to be about 28,000 (5.6 \% on average) [3]. This is significantly lower than the rate of the more developed EU member states [4]. According to expert opinion, access to biological treatments in other inflammatory conditions (Crohn's disease, ulcerative colitis and psoriasis) is even lower, but local data on epidemiology and the number of patients treated with biologicals are very limited. As the NICE definition pointed out very clearly, guidelines help healthcare professionals in their work, but they do not replace their local knowledge and skills when taking national priorities and values into consideration.
Biosimilars may improve access to expensive treatments to patients with inflammatory conditions, especially in the middle income countries. Biosimilar infliximab became available in CEE earlier and to a larger extent than in other countries. Experience gained in CEE might be useful to other countries as well, because CEE countries are early adopters of the first biosimilar anti-TNF drugs. A number of drugs are being developed currently to attain biosimilar status in inflammatory conditions [5]. One paper in this supplement discusses the statistical and regulatory considerations in the assessments of interchangeability of biologicals. Two other papers provide results from metaanalyses of clinical efficacy and safety of biosimilar infliximab compared to other biologicals in rheumatoid arthritis and ankylosing spondylitis.

When it comes to diffusion of biosimilars, biosimilar competition might differ from generic competition for various reasons. Experience with biosimilar drugs in other treatment fields confirmed the variability in biosimilar utilisations across countries. In a recent study, the biosimilar uptake of epoetin, filgrastim and somatotropin is very different in the $17 \mathrm{EU}$ countries, (no countries from CEE were involved) [6]. The percentage of sales in daily defined dose (DDD) of biosimilars of the total market varies greatly from country to country (epoetin: $1 \%$ in Switzerland and Ireland, $54 \%$ in Greece, $45 \%$ in Germany), filgrastim uptake is the highest in Austria and Norway (64\%) and only $2 \%$ in Denmark, somatotropin uptake is highest in Sweden and lowest in the UK (2\%) in the countries from where results are available and based on the data provided by IMS Health. Interpretation of these data is very difficult; however, these figures suggest that diffusion of biosimilar drugs is primarily driven by national regulation and financing decisions. Based on the same scientific evidence provided on biological drugs, these policies and financing decisions vary greatly.

One of the inputs for making reimbursement decisions that determines access for patients to the new expensive therapies comes from HTA. The aim of HTA is to support the formulation of safe, effective, health policies that are patient-focused and seek to achieve best value [7].

Health technology assessment has been implemented to some extent in this group of CEE countries but challenges remain in positioning it as part of national policy making. HTA guidelines set in most countries require a large amount of data, including disease burden evidence from clinical studies, patient reported outcome, results from health economics analyses, modelling and budget impact analyses, etc. However, there is a massive inconsistency between what is required and what is available in terms of data on epidemiology, costs, cost of illness, and costeffectiveness of the technology seeking public funding. HTA in CEE countries now seems to be at a crossroads. 
For further development of HTA, local data are increasingly needed as input for analyses and decisions which are grounded in national priorities and values [8].

In this supplement we describe and discuss the development and use of HTA in Poland, the Czech Republic, Hungary, Romania and Bulgaria.

Due to the shortage of locally conducted HTA reports and health economics analyses in these countries, the transferability of results from other HTA jurisdictions (mainly from NICE UK) has key importance. One of the papers provides some thoughts and practical approaches for transferring results from cost-utility analysis of biologicals in inflammatory conditions to the CEE jurisdictions.

In this supplement there are original papers in rheumatology discussing subjective health expectations at biological therapy initiation and evaluating acceptability of less than perfect health states in rheumatoid arthritis. Real life experience with switching anti-TNFs in ankylosing spondylitis is presented based on the analysis of a local registry. Other papers provide data on work disability and productivity loss in patients with inflammatory bowel diseases, cost-of-illness in patients with moderate to severe psoriasis and, moreover, on the mapping of EQ-5D utility scores on two disease-specific assessment tools (DLQI and PASI) in psoriasis.

The availability of biological and biosimilar drugs represents one of the most striking advances in medical science in health care in the past decade [9], the potentially achievable benefit is very high. However, our priorities are shifting and more and more attention is given to how to use what we already have and what we already know to produce better outcomes. The benefit achieved depends to a large extent on the quantity and quality of local data available on epidemiology, disease burden, quality of life, local values and the validity of HTA recommendations, the institutionalisation of HTA informed health policy and financing decisions.
Papers in this supplement provide useful data for policy makers, funders and medical professionals to improve the outcome of biological and biosimilar treatments in inflammatory conditions in a cost-effective way in middle income countries.

Conflict of interest The publication of this supplement was sponsored by the Center for Public Affairs Studies Foundation, Corvinus University, Budapest.

\section{References}

1. http://guidance.nice.org.uk/CG

2. Brekke, K.R., Dalen, D.M., Holmas, T.H.: Diffusion of pharmaceuticals: cross-country evidence of anti-TNF drugs. Eur. J. Health. Econ. (2013). doi:10.1007/s10198-013-0536-6

3. Péntek, M., Poór, G., Wiland, P., Olerajova, M., Brzosko, M., Codrenau, C., Brodszky, N., Gulácsi, L.: Biologic therapy in inflammatory rheumatic diseases: issues in Central and Eastern European countries. Eur. J. Health Econ. (2014). doi:10.1007/ s10198-014-0592-6

4. Laires, P.A., Exposto, F., Mesquita, R., Martins, A.P., CunhaMiranda, L., Fonseca, J.E.: Patients' access to biologics in rheumatoid arthritis: a comparison between Portugal and other European countries. Eur. J. Health Econ. 14, 875-885 (2013)

5. Dörner, T., Strand, V., Castañeda-Hernández, G., Ferraccioli, G., Isaacs, J.D., Kvien, T.K., Martin-Mola, E., Mittendorf, T., Smolen, J.S., Burmester, G.R.: The role of biosimilars in the treatment of rheumatic diseases. Ann. Rheum. 72(3), 322-328 (2013)

6. Farfan-Portet, M.I., Gerkens, S., Lepage-Nefkens, I., Vinck, I., Hulstaert, F.: Are biosimilars the next tool to guarantee costcontainment for pharmaceutical expenditures? Eur. J. Health Econ. 15(3), 223-228 (2014)

7. European Network for Health Technology Assessment (EUnetHTA) www.eunethta.hu

8. Gulácsi, L., Rotar, A., Niewada, M., Loblova, O., Rencz, F., Petrova, G., Boncz, I., Klazinga, N.: Health technology assessment in Poland, the Czech Republic, Hungary, Romania and Bulgaria. Eur. J. Health Econ. (2014). doi:10.1007/s10198-014-0590-8

9. Jönsson, B., Kobelt, G., Smolen, J.: Patient access to rheumatoid arthritis treatments. Eur. J. Health Econ. 8(Suppl 2), S33-S34 (2008) 